* Autor para correspondencia. Servicio de Cardiología, Hospital General Universitario de Ciudad Real, C/ Obispo Rafael Torija, s/n, 13005 Ciudad Real, España.

Teléfono: +34926278000, Ext.: 78757.

Correo electrónico: javierjimenezdiaz@wanadoo.es (J. Jiménez-Díaz). http://dx.doi.org/10.1016/j.acmx.2016.07.004

1405-9940/

( 2016 Instituto Nacional de Cardiología Ignacio Chávez. Publicado por Masson Doyma México S.A. Este es un artículo Open Access bajo la licencia CC BY-NC-ND (http: / / creativecommons.org/ licenses/by-nc-nd/4.0/).

\section{Miocardiopatía hipertrófica obstructiva y anemia: causa y consecuencia}

\section{Hypertrophic obstructive cardiomyopathy and anemia: Cause and effect}

\section{Introducción}

La miocardiopatía hipertrófica $(\mathrm{MCH})$ es la miocardiopatía hereditaria más frecuente. Está causada por mutaciones en los genes que codifican las proteínas sarcoméricas, y se caracteriza por una hipertrofia asimétrica del septo interventricular, y distintos grados de obstrucción dinámica del tracto de salida del ventrículo izquierdo (OTSVI) por el movimiento sistólico anterior (SAM) de la valva anterior mitral ${ }^{1}$.
Su diagnóstico se basa en la detección de un aumento del grosor de la pared del ventrículo izquierdo (VI) mediante cualquier modalidad de imagen; incluyendo también el fenotipo de la enfermedad, la presencia de fibrosis miocárdica, las anomalías morfológicas del aparato de la válvula mitral, la función anómala de la microcirculación coronaria y las anomalías electrocardiográficas ${ }^{1}$.

La identificación de la OTSVI es importante en el manejo de los síntomas, y en la evaluación del riesgo de muerte súbita cardíaca. Se sabe que existen causas reversibles que pueden aumentar el gradiente dinámico: fármacos vasodilatadores, disminución de la precarga, ejercicio vigoroso, anemia..., por lo que se deben evitar dichas causas. El tratamiento farmacológico de elección es el uso de betabloqueantes ${ }^{1,2}$. Presentamos el caso de un paciente con $\mathrm{MCH}$ obstructiva severa y anemia poco habitual.
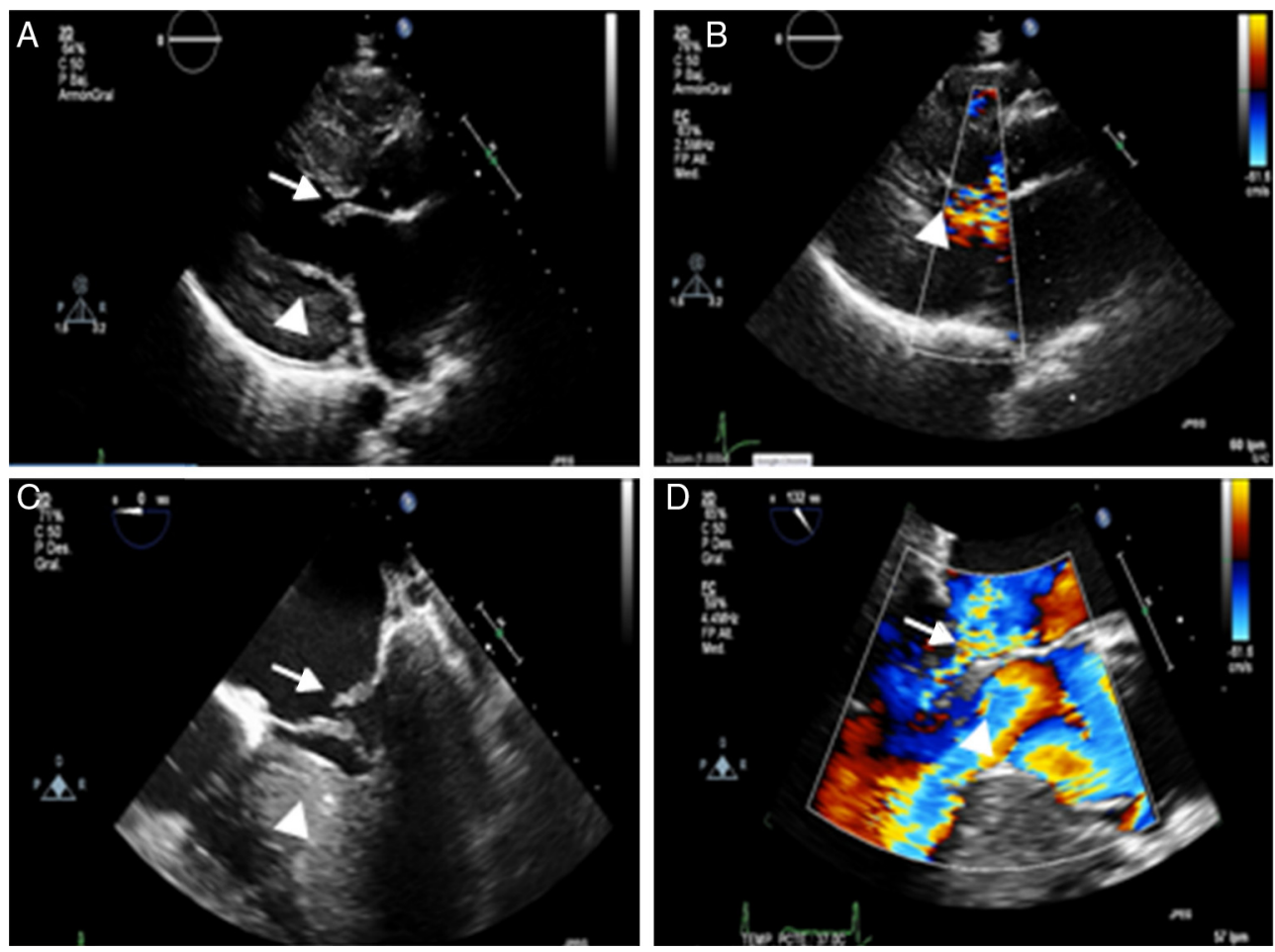

Figura 1 Ecocardiograma transtorácico (ETT) y transesofágico (ETE): A) Plano paraesternal eje largo se objetiva un vi con hipertrofia severa (punta de flecha) y diámetro disminuido, con velo mitral anterior algo engrosado (flecha). B) Plano paraesternal eje largo en sístole, con vi con colapso medioapical por la hipertrofia severa y con flujo turbulento tanto a nivel mitral como en el tracto de salida del vı (punta de flecha). C) ETE en proyección 4 cámaras donde destaca la hipertrofia ventricular (punta de flecha) con válvula mitral engrosada con prolapso y mala coaptación del velo posterior (flecha). D) ETE en plano 3 cámaras, con insuficiencia mitral severa (punta de flecha) y obstrucción dinámica en el tracto de salida (flecha). 

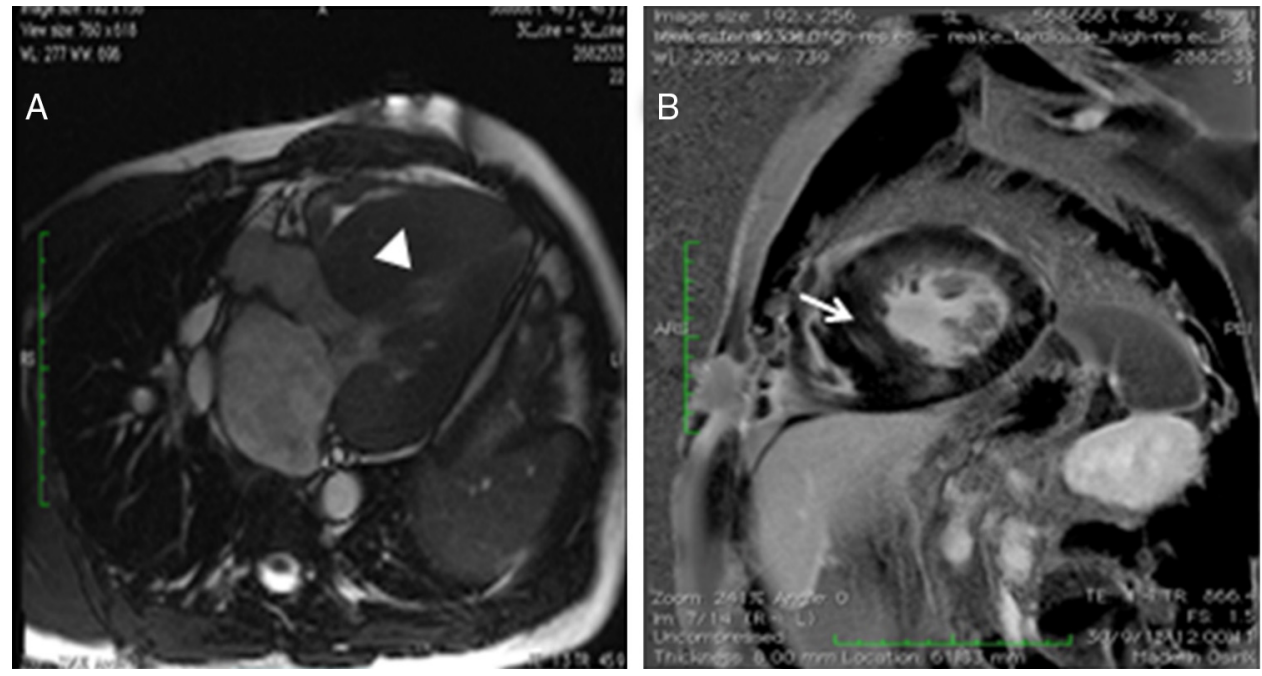

Figura 2 Resonancia magnética nuclear cardíaca: A) Corte axial potenciado en T2, donde se pone de manifiesto la importante hipertrofia ventricular (punta de flecha). B) Corte sagital con realce tardío donde se objetiva la hipertrofia y zonas de captación parcheada intramiocárdica difusa de predomino septal, lateral e inferior medio basal sugestivas de fibrosis (flecha).

\section{Caso clínico}

Varón de 48 años con $\mathrm{MCH}$ y anemia no estudiada en tratamiento domiciliario con hierro oral, que acude a nuestro hospital por dolor torácico y disnea. En la exploración física destacaba un murmullo vesicular conservado con crepitantes bibasales, unos ruidos cardíacos rítmicos con soplo holosistólico en foco mitral iv/vı que aumenta con maniobras de Valsalva, leve ictericia conjuntival, abdomen sin visceromegalias y episodios paroxísticos de orina de aspecto colúrico. En la ecocardiografía transtorácica (ETT) (fig. 1A y B) destacó hipertrofia concéntrica severa del VI, con espesor máximo del septo de $30 \mathrm{~mm}$, que generaba OTSVI con gradiente dinámico basal de $150 \mathrm{mmHg}$, y SAM que originaba insuficiencia mitral severa con jet excéntrico. En la analítica, al ingreso, destacaba una anemia normocítica ligera $(\mathrm{Hb} 11 \mathrm{~g} / \mathrm{dl}, \mathrm{VCM} 84 \mathrm{fl}$ ). Durante su estancia hospitalaria requirió del uso de betabloqueantes intravenosos para su manejo. Asimismo, se amplió estudio analítico, destacando una hemoglobina $10.5 \mathrm{~g} / \mathrm{dl}$, VCM $82.8 \mathrm{fl}$, reticulocitos $2.8 \%$, haptoglobina $<8 \mathrm{mg} / \mathrm{ml}, \mathrm{LDH} 2,309 \mathrm{U} /$, hierro $48.7 \mu \mathrm{g} / \mathrm{dl}$ y bilirrubina total $2.5 \mathrm{mg} / \mathrm{dl}$. Test de Coombs directo e indirecto negativo. Se realizó una sangre oculta en heces que resultó negativa. Ecografía abdominal dentro de los límites normales. Para completar el estudio se realizó una ecocardiografía transesofágica (ETE) (fig. 1B y C) en la que se observó un vi hiperdinámico, con hipertrofia concéntrica severa, un ventrículo derecho con hipertrofia de su pared libre $(8 \mathrm{~mm})$ y función sistólica conservada, la aurícula izquierda estaba dilatada de forma moderada con tendón paralelo al septo interauricular, obteniéndose un gradiente subaórtico dinámico basal severo $(115 \mathrm{mmHg})$. La válvula mitral estaba engrosada, con velo anterior grande, con una longitud de $34 \mathrm{~mm}$ y con un SAM que causaba prolapso y mala coaptación del velo posterior con una insuficiencia mitral severa. Se realizó RMN cardíaca (fig. 2A y B) compatible con miocardiopatía hipertrófica de predomino septal y anterior con grosor máximo de $33 \mathrm{~mm}$. Se demostró una captación patológica parcheada intramiocárdica difusa de predominio septal, inferior y lateral medio basal sugestiva de fibrosis. Se realizó cateterismo, que descartó la presencia de enfermedad coronaria con un árbol coronario sin lesiones. Durante su estancia hospitalaria se realizó registro Holter sin alteraciones destacables del ritmo y/o conducción. Destacó en la evolución empeoramiento clínico; coincidiendo con disminución de las cifras de hemoglobina $(9 \mathrm{~g} / \mathrm{dl})$, por lo que se decidió transfusión sanguínea y, dada la magnitud de la OTSVI a pesar de dosis máximas de metoprolol, traslado al servicio de cirugía cardíaca para realización de miomectomía septal y reparación valvular mitral, no pudiéndose llevar a cabo esta, por lo que se realizó sustitución valvular mitral por prótesis mecánica mitral St Jude $27 \mathrm{~mm}$ con buena evolución posterior.

La analítica de control, al mes de la intervención quirúrgica, mostró una tendencia a la normalización de los parámetros compatibles con anemia hemolítica: hemoglobina $14.5 \mathrm{~g} / \mathrm{dl}$, VCM $82.8 \mathrm{fl}$, reticulocitos $1 \%$, haptoglobina $11.5 \mathrm{mg} / \mathrm{ml}$, LDH $372 \mathrm{U} / \mathrm{l}$, hierro $84.9 \mu \mathrm{g} / \mathrm{dl}$ y bilirrubina normal. El ecocardiograma de control mostró hipertrofia vI, buena función ventricular sin gradiente dinámico en reposo ni tras Valsalva y con prótesis mecánica mitral normofuncionante (gradiente medio de $3.5 \mathrm{mmHg}$ ).

\section{Discusión}

La anemia hemolítica se ha descrito en el contexto de prótesis aórticas mecánicas y en estenosis valvular aórtica (síndrome de Heyde) ${ }^{3}$, pero raramente en asociación con $\mathrm{MCH}$. De hecho, la anemia se considera un factor a tener en cuenta en el empeoramiento clínico de los pacientes con MCH obstructiva, por aumento del gradiente dinámico; siendo la hemólisis mecánica una causa rara de anemia en estos pacientes. Se sabe que la hemólisis de los glóbulos rojos, en condiciones normales, se produce cuando estos se someten a un estrés en torno a 3,000 dinas $/ \mathrm{cm}^{2}$, incrementándose en gran medida por encima de dicho nivel. La 
OTSVI proporciona un aumento en la tensión y, por lo tanto, cizallamiento en los glóbulos rojos, pudiéndose desarrollar a partir de gradientes de presión mayores a $50 \mathrm{mmHg}$ (estrés 4,000 dinas $/ \mathrm{cm}^{2}$, superándose el límite de la membrana de resistencia a la tracción de los eritrocitos) ${ }^{4}$. Así, en algunos pacientes la OTSVI que aparece en la $\mathrm{MCH}$ puede conducir a anemia hemolítica por la hemólisis mecánica que sufren los eritrocitos al exponerse al flujo turbulento que se genera, pudiendo actuar además como precipitante de los cuadros de descompensación (por ser la anemia persé causa de aumento de gradiente dinámico $)^{5}$; generándose un círculo vicioso: OTSVI-anemia hemolítica-insuficiencia cardíaca.

\section{Conclusión}

Con este caso queremos reseñar que es fundamental filiar la causa, si estuviese presente, de la anemia en un paciente con $\mathrm{MCH}$, puesto que nos puede ayudar a la hora de plantear las posibilidades terapéuticas (miectomía septal) ${ }^{1}$; evitándose así caer en el círculo vicioso antes comentado. Es cierto que en la analítica de control todavía hay parámetros que no se han normalizado, pero es evidente que tras la cirugía el paciente presenta mejoría del síndrome anémico y tendencia a una normalización de dichos valores, por lo que consideramos la OTSVI como la causa fundamental de la anemia.

\section{Bibliografía}

1. Grupo de Trabajo de la ESC para el diagnóstico y manejo de la miocardiopatía hipertrófica. Guía de práctica clínica de la ESC 2014 sobre el diagnóstico y manejo de la miocardiopatía hipertrófica. Rev Esp Cardiol. 2015;68:63.e1-e52.
2. Montijano A, Zubeldía B, Penas M, et al. Estrategias terapéuticas en la miocardiopatía hipertrófica obstructiva sintomática. Rev Esp Cardiol. 2001;54:1311-26.

3. Massyn MW, Khan SA. Heyde syndrome: A common diagnosis in older patients with severe aortic stenosis. Age Ageing. 2009;38:267-70.

4. Nevaril CG, Lynch EC, Alfrey CP Jr, et al. Erythrocyte damage and destruction induced by shearing stress. J Lab Clin Med. 1968;71:784-90.

5. Kubo T, Kitaoka H, Terauchi $\mathrm{Y}$, et al. Hemolytic anemia in a patient with hypertrophic obstructive cardiomyopathy. J Cardiol. 2010;55:125-9.

Leydimar Anmad Shihadeh*, Alejandro Quijada-Fumero, María Facenda-Lorenzo, Francisco J. Poncela-Mireles, Ana Laynez-Carnicero y Antonio Trugeda-Padilla

Servicio de Cardiología, Hospital Universitario Nuestra Señora de Candelaria, Santa Cruz de Tenerife, Islas Canarias, España

* Autor para correspondencia. Servicio de Cardiología, Hospital Universitario Nuestra Señora de Candelaria, Carretera del Rosario número 145, 38010 Santa Cruz de Tenerife, Islas Canarias, España.

Correo electrónico: leydimar_12@hotmail.com

(L. Anmad Shihadeh).

http://dx.doi.org/10.1016/j.acmx.2016.06.008 1405-9940/

(c) 2016 Instituto Nacional de Cardiología Ignacio Chávez. Publicado por Masson Doyma México S.A. Este es un artículo Open Access bajo la licencia CC BY-NC-ND (http: / / creativecommons.org/ licenses/by-nc-nd/4.0/).

\section{Conexión anómala parcial de venas pulmonares, un reto diagnóstico en cardiología pediátrica}

\section{Partial anomalous pulmonary venous connection in children, a challenging diagnose in pediatric cardiology}

La conexión anómala parcial de venas pulmonares (CAPVP), acostumbra a suponer un reto diagnóstico en pediatría, pues la mayoría de pacientes se encuentran asintomáticos. Esta entidad suele ir asociada a comunicación interauricular, ya sea tipo ostium secundum o seno venoso ${ }^{1-3}$. Cuando se presenta de manera aislada, una dilatación de cavidades derechas detectada en una ecocardiografía puede ser la única pista diagnóstica.

\section{Breve exposición de 2 casos}

El primer caso se trata de una paciente adolescente afecta de una comunicación interauricular tipo ostium secundum que se cerró quirúrgicamente a los 5 años de vida. Sin otros antecedentes a destacar. En el seguimiento ecocardiográfico posterior, a partir del tercer año poscirugía se evidenció una dilatación progresiva de cavidades derechas. El diámetro telediastólico del ventrículo derecho alcanzó $2.7 \mathrm{~cm}$ en eje paraesternal largo (valor Z-score de +3.7), con una estimación de Qp/Qs de 1.7. La paciente se hallaba asintomática, y no se auscultaba soplo cardíaco a la exploración física. Al no lograrse identificar las venas pulmonares izquierdas, se sospechó de una CAPVP, y se pidió una angiotomografía computarizada (angio-TC) cardíaca, que confirmó el diagnóstico. La vena pulmonar superior izquierda se observó que drenaba a la vena innominada a través de una vena vertical, mientras que la vena pulmonar inferior izquierda drenaba a un seno coronario dilatado (fig. 1), tratándose pues de una CAPVP izquierda mixta (intracardíaca y supracardíaca). La paciente fue re-intervenida electivamente a los 14 años de edad.

El segundo caso se trata de una niña de 3 años que fue remitida a la consulta externa de cardiología pediátrica para valoración de soplo sistólico. Como antecedentes destacaban una bronconeumonía y varios episodios de bronquitis cada invierno. La ecocardiografía reveló un ventrículo derecho dilatado, con un diámetro telediastólico de $2.2 \mathrm{~cm}$ en 